\title{
Assessment of the ripple effects and spatial heterogeneity of total losses in the capital of China after a great catastrophic shock
}

\author{
Zhengtao Zhang ${ }^{1,3}$, Ning Li ${ }^{1,2,3}$, Wei Xie ${ }^{4}$, Yu Liu ${ }^{5}$, Jieling Feng ${ }^{1,3}$, Xi Chen ${ }^{1,3}$, and Li Liu ${ }^{1,3}$ \\ ${ }^{1}$ State Key Laboratory of Earth Surface Processes and Resource Ecology, Faculty of Geographical Science, \\ Beijing Normal University, Beijing 100875, China \\ ${ }^{2}$ Key Laboratory of Environmental Change and Natural Disaster, MOE, Faculty of Geographical Science, \\ Beijing Normal University, Beijing 100875, China \\ ${ }^{3}$ Academy of Disaster Reduction and Emergency Management, Ministry of Civil Affairs and Ministry of Education, \\ Faculty of Geographical Science, Beijing 100875, China \\ ${ }^{4}$ China Center for Agricultural Policy, School of Advanced Agricultural Sciences, Peking University, No. 5 Yiheyuan Road, \\ Haidian District, Beijing 100871, China \\ ${ }^{5}$ Institutes of Science and Development, Chinese Academy of Sciences, No. 15 Zhongguancun Beiyitiao, Haidian District, \\ Beijing 100190, China
}

Correspondence to: Zhengtao Zhang (zhangzhengtao@mail.bnu.edu.cn) and Ning Li (ningli@ bnu.edu.cn)

Received: 2 November 2016 - Discussion started: 14 November 2016

Revised: 4 February 2017 - Accepted: 13 February 2017 - Published: 9 March 2017

\begin{abstract}
The total losses caused by natural disasters have spatial heterogeneity due to the different economic development levels inside the disaster-hit areas. This paper uses scenarios of direct economic loss to introduce the sectors' losses caused by the 2008 Wenchuan earthquake (2008 WCE) in Beijing, utilizing the Adaptive Regional Input-Output (ARIO) model and the Inter-regional ripple effect (IRRE) model. The purpose is to assess the ripple effects of indirect economic loss and spatial heterogeneity of both direct and indirect economic loss at the scale of the smallest administrative divisions of China (streets, villages, and towns). The results indicate that the district of Beijing with the most severe indirect economic loss is the Chaoyang District; the finance and insurance industry (15, see Table 1) of Chaowai Street suffers the most in the Chaoyang District, which is 1.46 times that of its direct economic loss. During 2008-2014, the average annual GDP (gross domestic product) growth rate of Beijing was decreased $3.63 \%$ by the catastrophe. Compared with the $8 \%$ of GDP growth rate target, the decreasing GDP growth rate is a significant and noticeable economic impact, and it can be efficiently mitigated by increasing rescue effort and by supporting the industries which are located in the seriously damaged regions.
\end{abstract}

\section{Introduction}

Economic losses caused by frequent natural disasters have increased dramatically and pose serious challenges to the world's sustainable development and human safety (Munich Re, 2002). In April 2016, earthquakes with a surface wave magnitude $\left(M_{\mathrm{S}}\right)$ of 7 occurred within 8 days in succession in Japan $\left(M_{\mathrm{S}}=7.3\right)$, Ecuador $\left(M_{\mathrm{S}}=7.8\right)$, Burma $\left(M_{\mathrm{S}}=7.2\right)$, and Afghanistan $\left(M_{\mathrm{S}}=7.1\right)$, which caused large total economic losses to these countries. The large economic losses caused by natural disasters should be assessed more accurately to improve the awareness of disaster impacts and increase the effectiveness of disaster prevention and mitigation.

The total economic losses caused by disasters usually incorporate the direct economic loss (Algermissen and Steinbrugge, 1984; Lijun et al., 1998; Jiren et al., 2003; Ming et al., 2014) and the indirect economic loss. The direct economic loss is caused by the physical damage when the disaster occurs and the induced physical impact left after (Cochrane, 1997). The indirect economic loss is generated by business interruption, imbalance between supply and demand, disorder between forward output, and backward supply of sectors due to physical damage (Cochrane, 1997; 


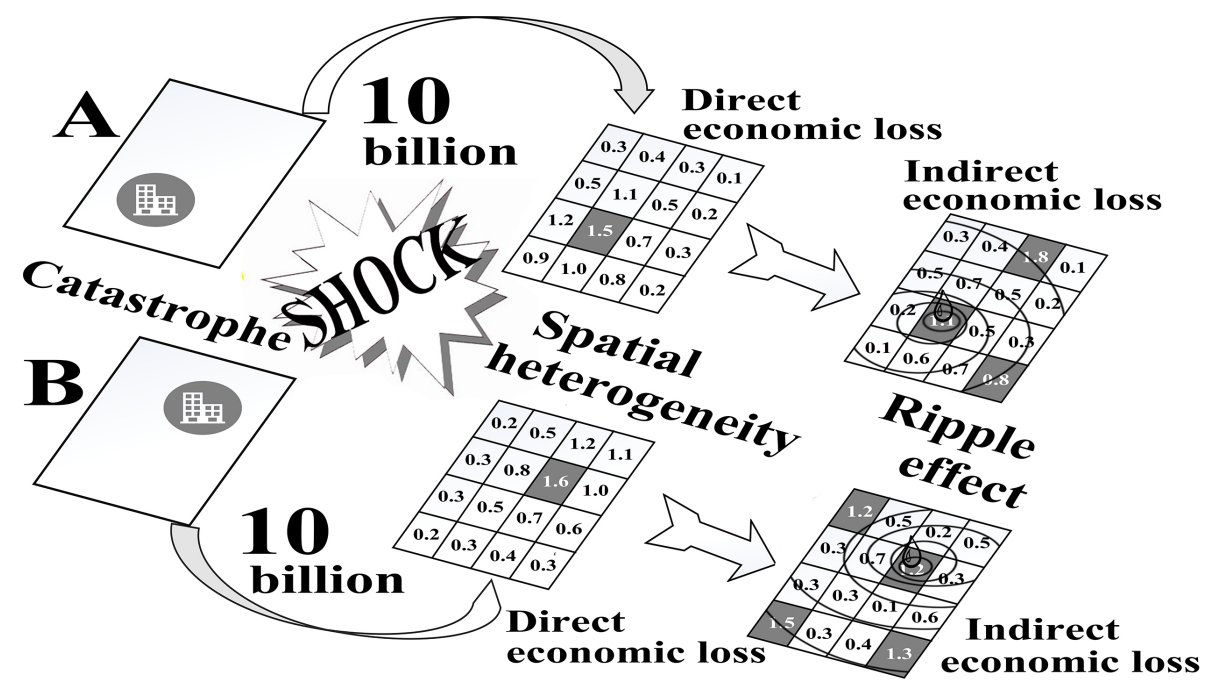

Figure 1. The diagrammatic representation of spatial heterogeneity and the ripple effects. A and B represent two disaster-hit areas with different functional zoning. If a catastrophe with the same intensity hits, both suffer a direct economic loss (DEL) of USD 10 billion, but the losses inside the areas are different due to functional zoning and the disaster's location. The ripple effects of the indirect economic loss (IEL) are not only impacted by the distance of the occurring disaster; they are also impacted by the interregional and interindustrial linkages.

FEMA, 2001). This property of "invisible loss" makes it difficult to evaluate, but it cannot be ignored. Because indirect economic loss can increase, it can even outnumber direct economic loss along with the economic development, which underscores the significance of being able to capture the ripple effects accumulated along interregional and interindustrial linkages. The Input-Output (IO) model (Hallegatte et al., 2010; Wu et al., 2012; Hallegatte, 2014; Zhou et al., 2014; Xia et al., 2016) and the computable general equilibrium (CGE) model (Rose and Liao, 2005; Guivarch et al., 2009; Xie et al., 2013; Rose, 2015) are two representative models which are commonly used to assess indirect economic loss.

However, most of the loss assessment methods only consider the overall loss value of the region (Xie et al., 2012); the losses in different regions inside the disaster-hit area have not yet been quantified. In a modern city, the functional zoning has its own economic characteristics because of the industrial distribution, so there may be only one or two developed economic sectors existing in each functional zone. If a catastrophe occurs, the direct economic losses of sectors will not be evenly distributed inside the disaster-hit area due to the range of disaster impacts (Fig. 1). In addition, as a result of the industrial linkage, the ripple effect of indirect economic loss can make a sector's loss spread to other related sectors in other regions (e.g., the production reduction of an automobile manufacturing sector increases the unintended inventory, or decreases production, of a steel sector in another region, eventually making its indirect economic loss increase) (Fig. 1). Therefore, there is obvious spatial heterogeneity in the direct and indirect economic losses of sectors inside the disaster-hit area. The distribution of total losses are inconsis- tent in two disaster-hit areas with a similar economic development degree (or whole total losses).

Much attention has been paid to assessing the total loss of the whole disaster-hit area, but further study on the refined assessment of direct economic loss and the ripple effect of indirect economic loss inside the area is crucial to revealing the comprehensive disaster impacts, though internal economic data are difficult to acquire and the assessment models are rare. The results of the above research will play a significant role in different aspects. For governments, policy makers can make more refined post-disaster recovery and reconstruction policies and give the sectors with closer industrial linkages priority to make the economic system recover to pre-disaster levels faster. For the insurance firms, they can add insurance categories to increase profits and also enhance the economic resilience of economic system. For the public, people can be fully aware of the long-term impact of disasters and thus change their economic activities (e.g., real estate investment).

The 2008 Wenchuan earthquake (2008 WCE) is designated as the catastrophe in this paper because it was the most destructive natural disaster since the founding of China in 1949. Beijing (BJ), one of the most important and developed metropolises in China, is chosen to be the disaster-hit area due to its location at the North China seismic belt (Fig. 2) and its prior experience with an earthquake (2 September 1679) of the same magnitude as the 2008 WCE. We introduce the sectors' direct economic losses caused by the 2008 WCE in BJ through this reasonable assumption.

This paper is the first to assess and analyze the ripple effects of indirect economic loss and spatial heterogeneity of 


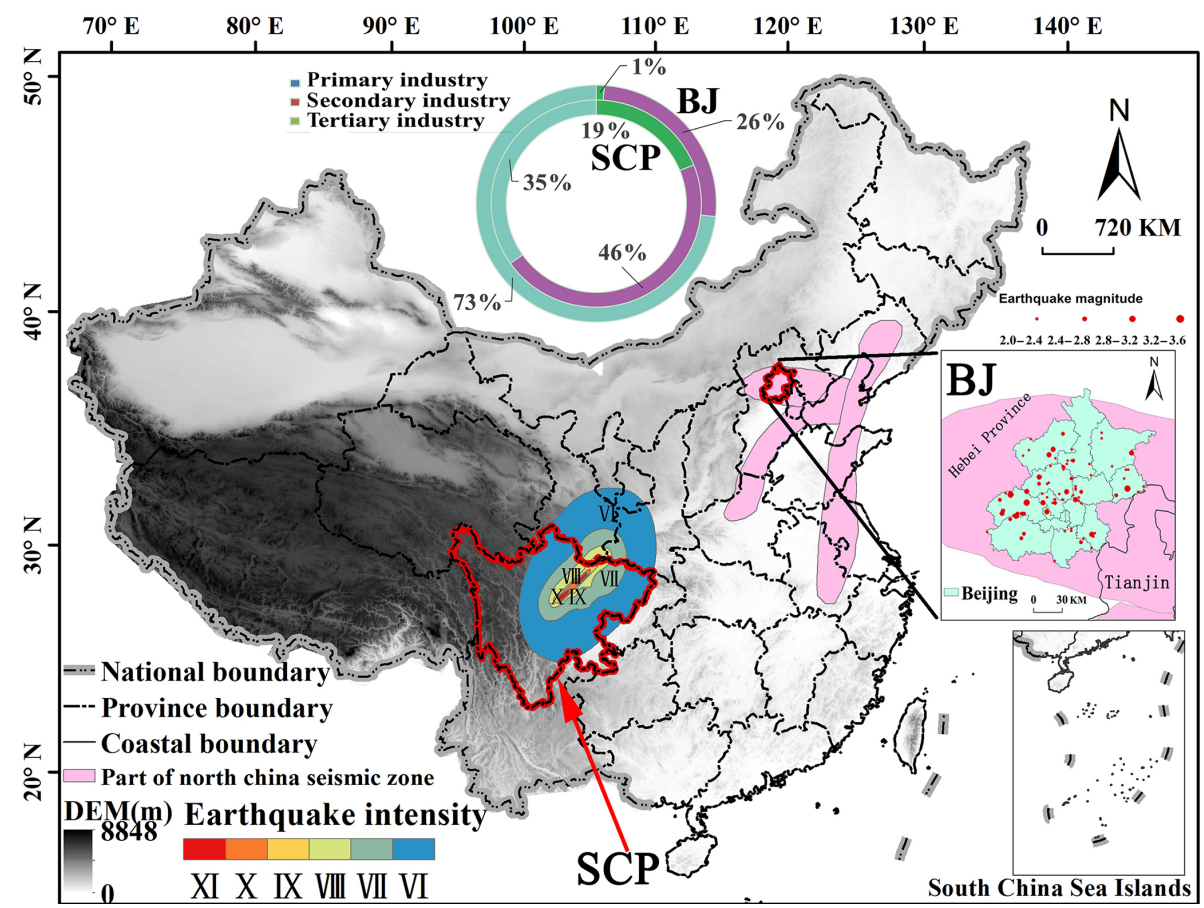

Figure 2. Sketch map of earthquakes in China and the locations of Beijing (BJ) and the Sichuan province (SCP). The top circle indicates the proportions of fixed asset stocks of primary, secondary, and tertiary industry in BJ (outer ring) and SCP (inner ring). The pink area around BJ shows the north China earthquake zone; the size of red points on the map of BJ indicate the earthquake magnitudes and locations.

both direct and indirect economic loss inside the disasterhit area at the scale of the smallest administrative divisions in China - streets, villages, and towns - by means of the Adaptive Regional Input-Output (ARIO) model (Hallegatte, 2008) and the Inter-regional ripple effect (IRRE) model.

This paper aims to decrease the long-term economic impacts caused by disasters and provide more comprehensive information for the government to improve post-disaster recovery, disaster prevention and mitigation, rescue measures, and fund allocation policies for the regions which may suffer serious damage in the disaster's aftermath.

\section{Study area and data}

\subsection{Study area}

The 2008 Wenchuan earthquake is chosen in this paper as the catastrophe because it is the earthquake with the greatest magnitude and the highest degree of damage since the founding of China in 1949; it affected 417 counties of 16 provinces and cities, leaving 69226 people dead and causing a direct economic loss of USD 124 billion (the exchange rate of CNY to USD was 0.14 in 2008). Out of all the affected provinces, the Sichuan province (SCP) suffered the most serious disaster with a direct economic loss of USD 104.9 billion, accounting for $89 \%$ of the total losses (NCDR and MOST, 2008). The Wu study (Wu et al., 2012) results show that in- direct economic loss of SCP caused by the earthquake was about USD 42.1 billion, which was $40 \%$ of its direct economic loss. The potential impact of the disaster on indirect economic loss has aroused wide concern from both the government and scholars (Rose et al., 1997; Okuyama, 2007; Li et al., 2013).

The occurrence of an earthquake in a city may lead to more economic losses (Tantala et al., 2008; Hancilar et al., 2010). Therefore, we chose Beijing (BJ) as the disaster-hit area. Between 2002 and 2014, BJ experienced 74 earthquakes with an $M_{\mathrm{S}}>2$, mainly concentrated in the Haidian District, the Changping District, and the Shunyi District as well as in the juncture between the Mentougou District and the Fangshan District (Fig. 2).

\subsection{Data}

The data used in this study are mainly divided into three parts.

1. Direct economic loss of BJ: the loss ratio of all sectors of SCP is taken as the scenario for the direct economic loss of BJ; the loss of SCP was acquired from the China National Commission for Disaster Reduction, a total of 17 sectors (NCDR and MOST, 2008). The specific calculation method is shown in Sect. 3.1. 
2. Input-output table: 42 sectors of input-output tables of BJ and SCP were acquired from the National $\mathrm{Bu}-$ reau of Statistics of the People's Republic of China. Since the input-output table is published once every 5 years in China (in the year ending with 2 and 7) and the 2008 WCE occurred in the middle of 2008 , it is proper to use the latest 2007 input-output table to represent the industrial linkage among sectors. In order to match the 2007 input-output table with the statistical loss data of 17 sectors which comes from the National Commission for Disaster Reduction, the 42 sectors are reconsolidated into 17 sectors in accordance with the Industrial classification for national economic activities (GB/T 4754-2011) and statistical data for the 2008 WCE.

3. Number of enterprises, their total fixed assets, and business incomes in 17 sectors within the smallest administrative divisions of streets, villages, and towns of $\mathrm{BJ}$; the data come from Beijing Macro Economic Social Development Basic Database. Because the time points of statistical data are not consistent, the administrative divisions of streets, villages, and towns are not consistent with the statistical data in the database and some streets, villages, and towns are consolidated, e.g., the Yizhuang region (including the national economic development zone).

\section{Research methods}

\subsection{Setting up the hypothetical scenario of direct economic loss of Beijing}

The reason for setting this scenario is that there is no direct economic loss in 17 sectors of Beijing (BJ) due to no real disaster occurring. In order to solve this problem, the scenario is set with an earthquake with the same intensity as the 2008 Wenchuan earthquake occurring in BJ and with BJ having the same direct economic loss ratio in 17 sectors as that of the Sichuan province (SCP). Before setting up this scenario, the economic conditions of the two areas should be understood.

\section{(1) Comparison of development between BJ and SCP}

In terms of social and economic development degrees, SCP was categorized as a less developed region, its GDP (gross domestic product) was USD 175.1 billion (calculated based on 2008 constant price, the same below). BJ was categorized as a developed region, its GDP in 2008 was USD 146.8 billion and almost equivalent to the GDP of the whole province of SCP. Meanwhile, in 2008, the per Capita GDP of BJ was 4.31 times that of SCP and the fixed asset stocks were 2.09 times greater. The per capita GDP of SCP only ranks 24 th of the 34 ranked provinces and cities of
China, while the GDP of BJ ranks second and is almost more than double the per capita GDP of an upper-middle-income country (USD 5511 in 2008, from World Bank). The population density of SCP is $168 \mathrm{~km}^{-2}$, which ranks $25 \mathrm{th}$, and that of $\mathrm{BJ}$ is up to $1079 \mathrm{~km}^{-2}$, which ranks 4 th. The proportion of the tertiary industry of BJ reached $73 \%$. Therefore, the developed secondary industry and tertiary industry as well as closer industrial linkage that may lead to a larger indirect economic loss is worthy of attention.

\section{(2) Scenario of direct economic loss}

The core assumption of the direct economic loss scenario is that both areas have the same sector loss rates. The core formula of the scenario is calculated as follows:

$$
\begin{aligned}
\operatorname{DEL}(\mathrm{BJ}, n, 2008) & =\frac{\operatorname{DEL}(\mathrm{SCP}, n, 2008)}{\operatorname{CAP}(\mathrm{SCP}, n, 2008)} \\
& \times \operatorname{CAP}(\mathrm{BJ}, n, 2008),
\end{aligned}
$$

where DEL (SCP or BJ, $n, 2008$ ) is the direct economic loss of the $n$th sector of SCP (or BJ) after the catastrophe, and CAP (SCP or BJ, $n, 2008$ ) is the fixed asset stock of the $n$th sector of SCP (or BJ) in 2008. The fixed assets stocks were calculated by the perpetual inventory method (Hall and Jones, 1999) according to the total investment in fixed assets of the whole society.

This method can visually reflect the shock of the 2008 WCE on BJ under the scenario of the same sector's loss ratio, and it can reduce the calculation uncertainty of direct economic loss caused by different economic development degrees between two regions. The results are illustrated in Table 1. It can be seen that the economic pattern of BJ was mainly dominated by the tertiary industry, while SCP was dominated by the secondary industry and was in an industrial transformation period.

\section{(3) Setting up the damage intensity scenarios of BJ}

It can be seen from Fig. 2 that the scope of the areas with a seismic intensity higher than IX degree covers all districts and counties of BJ. Furthermore, due to a lack of real loss data from BJ, if we add the seismic source location and the different seismic intensity ranges, the simulation uncertainty will be greatly increased. Therefore, we assume that the intensity of disaster damage is evenly distributed in the space, and the neither seismic source location nor the intensity attenuation range is set. The upper limit of the losses caused by a violent earthquake in $\mathrm{BJ}$ is given through such extreme assumption.

\subsection{Setting rescue scenarios}

After a catastrophe, the Chinese government's rescue policy is "concentrating the whole country's efforts on rescue at all costs" (Editorial department of Chinese geological education, 2008), the rescue and reconstruction are led 
Table 1. Calculation results of the stock of fixed assets and the direct economic loss of BJ and SCP.

\begin{tabular}{|c|c|c|c|c|c|c|c|}
\hline \multirow[t]{2}{*}{ ID } & \multirow[t]{2}{*}{ Sectors } & \multirow[t]{2}{*}{ Industry } & \multicolumn{2}{|c|}{ Fixed asset stocks } & \multirow{2}{*}{$\begin{array}{l}\text { Loss } \\
\text { ratio }\end{array}$} & \multicolumn{2}{|c|}{ DEL } \\
\hline & & & $\mathrm{SCP}$ & $\mathrm{BJ}$ & & $\mathrm{SCP}$ & BJ \\
\hline 1 & Agriculture & 1 & 491.7 & 13.93 & 0.03 & 16.80 & 0.48 \\
\hline 2 & Mining industry & 2 & 138.69 & 18.73 & 0.10 & 14.00 & 1.89 \\
\hline 3 & Food manufacturing and tobacco processing & 2 & 138.61 & 28.97 & 0.18 & 25.20 & 5.27 \\
\hline 4 & Textile manufacturing & 2 & 34.65 & 10.71 & 0.18 & 6.30 & 1.95 \\
\hline 5 & Wood processing and furniture manufacturing & 2 & 34.65 & 23.09 & 0.18 & 6.30 & 4.20 \\
\hline 6 & Coke, gas, and oil processing & 2 & 10.40 & 31.24 & 0.08 & 0.84 & 2.52 \\
\hline 7 & Chemical industry & 2 & 97.03 & 68.33 & 0.18 & 17.50 & 12.32 \\
\hline 8 & Nonmetallic mineral manufacturing & 2 & 41.58 & 17.81 & 0.18 & 7.56 & 3.24 \\
\hline 9 & Metallic products manufacturing & 2 & 103.96 & 52.06 & 0.18 & 18.20 & 9.11 \\
\hline 10 & Mechanical equipment manufacturing & 2 & 173.27 & 263.85 & 0.18 & 30.80 & 46.90 \\
\hline 11 & Electricity, steam, and hot-water production and supply & 2 & 623.59 & 265.27 & 0.18 & 112.00 & 47.64 \\
\hline 12 & Building trade & 2 & 32.96 & 30.98 & 0.11 & 3.50 & 3.29 \\
\hline 13 & Transportation, post, and telecommunications & 3 & 708.67 & 872.57 & 0.17 & 117.60 & 144.80 \\
\hline 14 & Commerce and catering & 3 & 78.10 & 89.83 & 0.16 & 12.60 & 14.49 \\
\hline 15 & Finance and insurance & 3 & 1082.59 & 869.16 & 0.14 & 154.00 & 123.64 \\
\hline 16 & Specific service management & 3 & 126.37 & 428.71 & 0.07 & 8.4.0 & 28.50 \\
\hline 17 & Other services & 3 & 425.80 & 562.73 & 0.18 & 77.00 & 101.76 \\
\hline
\end{tabular}

Note: (1) unit is USD 0.1 billion, calculated based on 2008 constant price; (2) SCP is the Sichuan province, BJ is Beijing, and DEL is the direct economic loss.

Table 2. ARIO model parameters and parameter settings of different post-disaster rescue scenarios for Beijing (BJ).

\begin{tabular}{llllll}
\hline & & & \multicolumn{2}{c}{ Scenario B } \\
\cline { 4 - 5 } Parameter & Description & Scenario A & Scenario B1 & Scenario B2 & Scenario C \\
\hline$a_{b}$ & Production capacity pre-disaster & $100 \%$ & $100 \%$ & $100 \%$ & $100 \%$ \\
$a_{\max }$ & Maximum production capacity post-disaster & - & $120 \%$ & $120 \%$ & $150 \%$ \\
$\tau$ & Adaptation time & - & 6 months & 3 months & 3 months \\
$\xi$ & Price elastic coefficient & 0.9 & 0.9 & 0.9 & 0.9 \\
\hline
\end{tabular}

by the central government, which is not dependent on the market (e.g., insurance). For the recovery and reconstruction work for the 2008 WCE, the Chinese government provided $120 \%$ of the maximum production capacity, compared to pre-disaster capacity, through rescue efforts (NCDR and MOST, 2008). Considering the of importance of BJ and its degree of economic development, if a devastating earthquake occurs, rescue efforts may make the maximum production capacity reach $150 \%$, compared to pre-disaster capacity, and the recovery period may be shorter. For this purpose, three rescue efforts scenarios are set and illustrated in Table 2. The price elastic coefficient parameter is 0.9 (Wu et al., 2012; Xie et al., 2012). By comparing the indirect economic losses of BJ under different rescue scenarios, the necessity of improving rescue efforts is evaluated.

- Rescue scenario A: a natural recovery only satisfying the demand of basic disaster relief and depending on BJ's own economic structure and industrial linkage to make the economic system recover to its pre-disaster level.
- Rescue scenario B: BJ is provided with $120 \%$ of its maximum production capacity, compared to its predisaster capacity, according to the real rescue policy for the 2008 WCE. Scenario B1 is the maximization of production capacity accomplished within 6 months, and scenario B2 is the improvement of the production capacity to maximum 3 months ahead of schedule of Scenario B1.

- Rescue scenario $\mathrm{C}$ : $\mathrm{BJ}$ is provided with stronger rescue efforts $(150 \%)$ and a shorter production capacity improvement period (3 months), considering the economic development degree, position, and importance of BJ.

\subsection{The assessment model of indirect economic loss}

This paper utilized the Adaptive Regional Input-Output model to dynamically assess the indirect economic loss. The ARIO model was proposed by Hallegatte, and it was successfully applied to the assessment of indirect economic loss caused by the 2005 Hurricane Katrina (Hallegatte, 2008, 2014); floods in Mumbai, India (Ranger et al., 2010); and 
the 2008 WCE (Wu et al., 2012). This model is based on the traditional IO model and combines some advantages of the CGE model. The CGE model considers many complicated factors based on nonlinear assumptions such as market feedback and price change, but it also demands detailed statistical data and a complicated process of parameter validation. These data are difficult to acquire at the "urban" scale and even at the "streets, villages, and towns" scale. Therefore, the ARIO model is appropriate in this study, as it takes a full consideration on economic characteristics after the disaster - such as the change of production capacity of various economic sectors, production bottleneck, and capacity restriction caused by the impact of production reduction, production halts, and industrial linkage between various sectors to make a dynamic simulation of the change in the balance of supply and demand of the economic system in the time period from the catastrophe's occurrence to the completion of reconstruction. This describes the impact of the disaster in the regional economy. The modeling time step width is 1 month. The key parameters of the model (Table 2) and the core formula are illustrated as follows:

$$
\begin{aligned}
Y(j) & =\overbrace{\sum_{j} A(i, j) Y(j)}^{\text {intermediate demand }} \\
& +\overbrace{\operatorname{LFD}(i)+E(i)+\operatorname{HD}(i)+\sum_{j} D(j, i)}^{\text {final demand }} .
\end{aligned}
$$

The parameter on the left side of the formula is the total output $(Y(j))$, and parameters on the right side of the formula represent the demand, including intermediate demand and final demand. The cascading impact of the disaster on the economic system is reflected in the change in demand (intermediate demand and local final demand, LFD) within the disaster-hit areas and change in imports $(E)$, the increase the demands of households (HD) and commercial sectors $(D)$, and the reduction $(D)$ in production capacity caused by the reduction of capital.

In order to better understand the ARIO model, some key parameters or processing steps are explained. The ARIO model copes with the imports part in three ways. (i) First, by improving the traditional IO table to the Local Input-Output (LIO) table based on the coefficient $P_{j}$, $P_{j}=($ Production - Export $) /($ Production - Export + Import $)$. The model multiplies the values of quadrant I and quadrant II of the traditional IO table by coefficient $P_{j}$ so as to remove the import part from the production and services of intermediate consumption and final consumption. (ii) Second, by setting the substitutability of sectors; the model sets the substitutability of sectors based on whether the sectors' production and services can be imported from outside of the disaster-hit area. In this study, the manufacturing sectors (3-10), the construction sector (12), and the transportation, post, and telecommunications sector (13) cannot be substituted; the other sectors' productions and services can be imported from outside areas. (iii) Finally, the ARIO model imports changes during the reconstruction period; if a sectors can be substituted and its production capacity is insufficient, then the import part is introduced. The import amount depends on the relationship between demand and production, the parameter of import delay. The specific formula is as follows:

$$
I(j)+\frac{\operatorname{TD}^{t}(i)-Y(i)}{\operatorname{TD}^{t}(i)} A(j, i) \frac{\Delta t}{t_{A}^{\downarrow}} \stackrel{\Delta t}{\longrightarrow} I(j) .
$$

The sector $j$ is the sector that consumes the goods or services produced by sector $i, \mathrm{TD}(i)$ and $Y(i)$ are the demand and production of sector $i, A$ is the intermediate consumption, $\Delta t$ is the time step of model, $t_{A}^{\downarrow}$ is the parameter of import delay, and the initial $I(j)$ is the import of sector $j$ at the $t$ th month during the reconstruction period, which is decided by the ratio of the production of the $t$ th month and the predisaster production. The formula is shown as follows:

$I(t+1, j)=I(t, j) \times \frac{Y(t+1, j)}{Y(1, j)}$.

All these steps are calculated based on the LIO table and the dynamic changes of the model; therefore, the imports factor is an endogenous variable.

The change in price is also an endogenous variable, calculated in this study by the relationship between demand and production in the disaster aftermath. The ARIO model assumes that commodity prices respond linearly to production and uses a single parameter of the price inflation variable (parameter $\xi$ in Table 2) for the economic system to apply these prices. Therefore, the change in prices do not strongly feed back into the simulation results. However, the Chinese government will strictly control the changes in price, avoiding serious inflation in the disaster aftermath. As a result, these assumptions agree with the characteristics of the rescue system of the Chinese government after the catastrophe. The price inflation variable is exogenous because it changes with the different economic systems of disaster-hit areas.

\subsection{The spatial diffusion (SDN) model of direct economic loss}

Direct economic loss is mainly caused by the damage to fixed assets belonging to inventory change. And as mentioned in Sect. 3.1, this study assumes that the intensity of disaster damage is evenly distributed in the areas of Beijing. Therefore, by utilizing the stock of fixed assets data of the streets, villages, and towns of $\mathrm{BJ}$, the post-disaster direct economic loss can be spatially extended into 321 street, village, and town areas. The core of formula of the SDN model is as follows: 
$\operatorname{DEL}_{\text {str }}(p)=\frac{\operatorname{CAP}_{\text {str }}(p)}{\operatorname{CAP}_{\mathrm{BJ}}} \times \operatorname{DEL}_{\mathrm{BJ}}(p=1,2,3 \ldots n)$,

where $\operatorname{DEL}_{\mathrm{str}}(p)$ is the direct economic loss of the $p$ th street of $\mathrm{BJ}, \mathrm{CAP}_{\mathrm{str}}(p)$ stands for the stock of fixed assets of the $p$ th street, $\mathrm{DEL}_{\mathrm{BJ}}$ stands for the direct economic loss, $\mathrm{CAP}_{\mathrm{BJ}}$ stands for the stock of fixed assets, and $n$ stands for 321 streets, villages, and towns in BJ. The stock of fixed assets is used in the SDN model because (i) it belongs to the concept of "stock", which is in line with the nature of direct economic loss, and the replacement costs of the stock of fixed assets account for the most of the statistical components of direct economic loss; and (ii) the core idea of the feedback of the production capacity of the economic system in the ARIO model is that the production capacities of sectors hit by the disaster decrease $x \%$ when the stocks of fixed assets decrease $x \%$. Therefore, using the sectors' stocks of fixed asset not only agrees with the statistical significance of direct economic loss, but also with the simulated mechanism of the ARIO model.

\subsection{The Inter-regional ripple effect (IRRE) model of indirect economic loss}

Indirect economic loss is mainly caused by the production reduction and industrial linkage of various economic sectors belonging to flow change. Therefore, according to business income of sectors of streets, villages, and towns, the ripple effects among sectors can be assessed at 321 streets, villages, and towns. The core of formula of the IRRE model is shown as the follows:

$$
\begin{array}{r}
\operatorname{IEL}_{\mathrm{str}}\left(p, s, \alpha_{\mathrm{max}}\right)=\frac{\operatorname{BIN}_{\mathrm{str}}(p, s)}{\operatorname{BIN}_{\mathrm{BJ}}(s)} \times \operatorname{IEL}_{\mathrm{BJ}}\left(s, \alpha_{\max }\right), \\
\left(p=1,2,3 \ldots n ; \alpha_{\max }=100,120,150 \%\right),
\end{array}
$$

where IEL $_{\text {str }}$ stands for the indirect economic loss of streets, villages, and towns; $\mathrm{BIN}_{\mathrm{str}}$ stands for business income of streets, villages, and towns; $\mathrm{BIN}_{\mathrm{BJ}}$ and $\mathrm{IEL}_{\mathrm{BJ}}$ stands for the business income and indirect economic loss of BJ respectively; $p$ stands for the $p$ th street; $s$ stands for the $s$ th sector; and $\alpha_{\max }$ stands for the three different rescue effort scenarios. The business income is used in the IRRE model because (i) the business income is the flow data, which is in line with concept of "flow" of the indirect economic loss; and (ii) in the earthquake disaster aftermath, the production reduction due to the limitation of production capacity, the production bottleneck, and the industrial linkage can be reflected in the business income of the affected year. The business income of a sector will be greatly affected if that sector suffers a large indirect economic loss. Therefore, it is reasonable to use business income in the IRRE model.

Former studies could only obtain the direct and indirect economic loss of a whole region, without more specific loss information inside the region. Using the established IRRE
Table 3. Evaluation results of the indirect economic loss of Beijing (BJ) under different rescue scenarios.

\begin{tabular}{lccccc}
\hline $\begin{array}{l}\text { Rescue } \\
\text { scenarios }\end{array}$ & $\begin{array}{c}\text { DEL* } \\
\text { (billion } \\
\text { USD) }\end{array}$ & $\begin{array}{c}\text { IEL* } \\
\text { (billion } \\
\text { USD) }\end{array}$ & $\begin{array}{c}\text { DEL*/ } \\
\text { Total } \\
\text { loss }\end{array}$ & $\begin{array}{c}\text { IEL*/ } \\
\text { Total } \\
\text { loss }\end{array}$ & $\begin{array}{c}\text { IEL*/ }^{*} \\
\text { DEL }^{*}\end{array}$ \\
\hline Scenario A & 88.1 & 62.5 & $58 \%$ & $42 \%$ & $71 \%$ \\
Scenario B1 & 88.1 & 41.2 & $68 \%$ & $32 \%$ & $47 \%$ \\
Scenario B2 & 88.1 & 37.1 & $70 \%$ & $30 \%$ & $42 \%$ \\
Scenario C & 88.1 & 17.5 & $83 \%$ & $17 \%$ & $20 \%$ \\
\hline
\end{tabular}

* Note: DEL is the direct economic loss.

model, we can not only accurately spread the post-disaster losses from the whole city to districts (streets, villages, and towns) which not only helps us understand the spatial heterogeneity of the losses, but we can also can propose "uneven" post-disaster recovery measures by analyzing the relationship between the direct and indirect economic loss of sectors in various streets to help the government determine the optimal recovery and reconstruction solutions for different areas by choosing to either increase rescue efforts to improve production capacity or by importing substitute products from outside, so as to achieve a quicker recovery to the post-disaster level.

\section{Results of loss assessment}

\subsection{Assessment results of the overall losses under the rescue scenarios}

According to the results of the simulation under an extreme scenario, Beijing (BJ) is likely to suffer USD 150.6 billion (the exchange rate of CNY to USD was 0.14 in 2008) of total loss, including USD 88.1 billion of direct economic loss and USD 62.5 billion of indirect economic loss (Table 3). The indirect economic loss accounts for $41.5 \%$ of the total loss and is almost the same as the indirect economic loss. The total loss has exceeded $2.6 \%$ of BJ's GDP in 2008 and accounts for $3.6 \%$ of the national GDP of China in 2008. During the 6 years after the catastrophe occurred (2008-2014), the average annual GDP growth rate of BJ could be decreased from 8.55 to $4.91 \%$, the value of decrease was up to $3.63 \%$; however, the goal for GDP growth rate of BJ was $8 \%$ and, therefore, the economic impact caused by the catastrophe seriously impeded the sustainable development in the future (Table 4).

Table 3 shows that, along with the intensifying rescue efforts, the indirect economic loss decreases gradually and thus the total losses decrease. When rescue efforts intensify from rescue scenario A (natural recovery) to rescue scenario $\mathrm{C}$ ( $150 \%$ of rescue efforts), the indirect economic loss is reduced by USD 45.1 billion, and the percentage of indirect economic loss to total losses is reduced from 42 to $17 \%$. By 
Table 4. The comparison of the GDP growth rate between no catastrophe and the assessment result under scenario A 6 years after the catastrophe occurred.

\begin{tabular}{lrrrrrrr}
\hline GDP growth rate & 2009 & 2010 & 2011 & 2012 & 2013 & 2014 & Average \\
\hline No catastrophe & $10.2 \%$ & $10.3 \%$ & $8.1 \%$ & $7.7 \%$ & $7.7 \%$ & $7.3 \%$ & $8.55 \%$ \\
Catastrophe occurred & $-1.1 \%$ & $4.3 \%$ & $5.3 \%$ & $6.5 \%$ & $7.3 \%$ & $7.2 \%$ & $4.92 \%$ \\
\hline
\end{tabular}

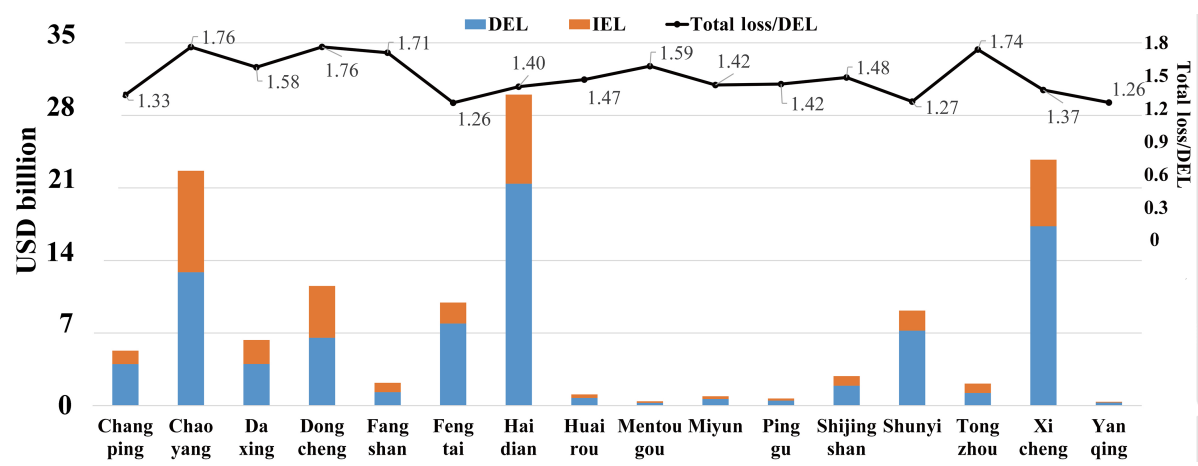

Figure 3. The direct and indirect economic loss of the districts of Beijing (BJ), and the ratios between total loss and direct economic loss. (Note: DEL is the direct economic loss, and IEL is the indirect economic loss.)

comparing the value of "IEL / DEL", it can be seen that when BJ suffers USD 100 billion of direct economic loss, its indirect economic loss is reduced from USD 71 billion in rescue scenario A to USD 20 billion in rescue scenario $C$.

Where does the reduction USD 51 billion in indirect economic loss come from? We can figure this out by further analyzing the reasons. After a disaster, damage to fixed assets leads to the inevitable reduction of production capacity, and the impact of industrial linkage on production capacity could be large or small. However, BJ is featured with strong industrial linkage, which brings a difficult economic recovery, long recovery period, and slow capital accumulation. Under the same rescue efforts, when the time for improving production capacity to maximization is 3 months ahead of schedule (scenario from B1 to B2), the indirect economic loss is reduced by USD 5 billion; under the same recovery period of 3 months, when rescue efforts increase by $30 \%$ (scenario from $\mathrm{B} 2$ to $\mathrm{C}$ ), the indirect economic loss is reduced by USD 22 billion.

In conclusion, rescue efforts play an important role in the post-disaster recovery and reconstruction. It is necessary to study how to properly use and scientifically allocate rescue and recovery funds according to the losses of different regions and different economic sectors. Therefore, the study will conduct an in-depth comparative analysis of the ripple effects of indirect economic loss and the spatial heterogeneity of direct economic loss in the next section.

\subsection{Spatial heterogeneity of direct economic loss and the ripple effects of indirect economic loss}

The losses are closely associated with the economic development of various regions of $\mathrm{BJ}$ and are not evenly distributed. If rescue funds are evenly distributed according to the total losses without considering the spatial differences of specific losses, it is likely to cause an improper allocation of funds and a reduction in rescue efficiency, which is not conductive to post-disaster recovery and reconstruction.

\section{(1) Differences in direct and indirect economic loss for the various districts}

Statistical loss data on the spatial scales of districts and counties are based on those of streets, villages, and towns, which can be seen in a macro perspective (Fig. 3). The three administrative districts with the most serious direct economic loss are Haidian District (USD 21.4 billion), Xicheng District (USD 17.3 billion), and Chaoyang District (USD 12.9 billion), while the three administrative districts with the most serious indirect economic loss are Chaoyang District (USD 9.8 billion), Haidian District (USD 8.6 billion), and Xicheng District (USD 6.4 billion). Among these, the indirect economic loss of the Chaoyang District accounts for $76 \%$ of its direct economic loss, and the total economic losses are 1.76 times that of its direct economic loss. Besides, although the indirect economic loss of the Chaoyang District is less than that of the Haidian District, its indirect economic loss is USD 1.2 billion higher than that of the Haidian District due to its developed tertiary industry and strong industrial linkages, demonstrating that indirect economic loss is rela- 


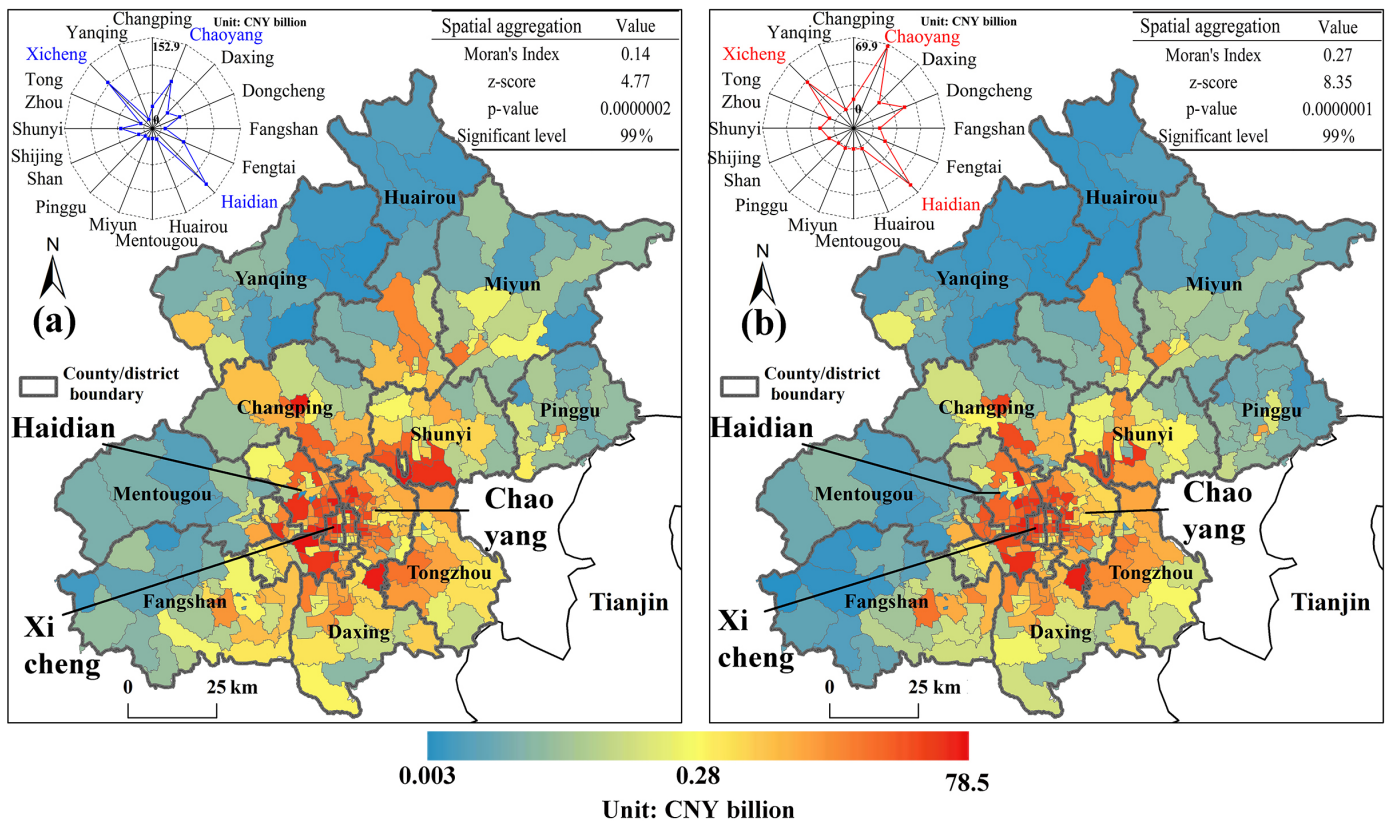

Figure 4. Spatial heterogeneity of the direct economic loss (a) and the ripple effects (b) of the indirect economic loss. The rose diagram in the upper left-hand corner of the figure represents the loss values of the districts. The table in the upper right-hand corner of figure represents the results of the spatial aggregation calculated using the spatial autocorrelation (Moran's I) method; the global Moran's I is an index used to measure spatial correlation, which is more obvious when its value is closer to 1 . The $z$ score and $p$ values are used to judge the confidence coefficient level; the results show that both of the spatial aggregations of DEL and IEL exceed the $99 \%$ confidence interval.

tively higher in economically developed regions and should not be ignored.

In addition to the ratio of total loss and direct economic loss in the Chaoyang District reaching 1.76, the ratios in the Dongcheng District, the Fangshan District, and the Tongzhou District are also higher than 1.70. The total losses of the Fangshan District and the Tongzhou District are relatively lower due to their relative poor economic development. According to the current industry structure and development planning, the government will ensure the establishment of sufficient disaster prevention and mitigation plans as well as recovery and reconstruction strategies in case of a future catastrophe occurrence.

\section{(2) Spatial heterogeneity of direct and indirect economic loss}

It can be seen from Fig. 4, firstly, that direct and indirect economic loss are mainly concentrated in main urban areas (Dongcheng District, Xicheng District, Haidian District, Chaoyang District, Shijingshan District, and Fengtai District). The longer the distance to the main urban areas is, the smaller the losses will be. Secondly, according to the spatial distribution, even though the indirect economic loss is smaller than the direct economic loss, the indirect economic losses of some streets, villages, and towns are higher than their corresponding direct economic losses. Therefore, the results show that even though the indirect economic loss of a region is smaller than direct economic loss, in some places inside the region it may greater than the direct economic loss. The results can help the government confirm the important rescue locations with more clarity. Thirdly, according to the spatial aggregation degree (Fig. 4), the spatial aggregation of the direct economic loss is 0.14 (Moran's index), and that of the indirect economic loss is 0.27 (Moran's index); both are significant at the $99 \%$ significance level. Therefore, the spatial distribution of high indirect economic loss values is more concentrated in the city center, while the distribution of high direct economic loss values is relatively dispersed.

The reasons may include the following. (1) The direct economic loss is mainly generated by the damage to fixed assets and, according to the spatial structural plan of BJ of "twoaxes, two-belts, and multiple centers" (Song, 2009), most of the traditional manufacturing enterprises have been moved outside the city center; therefore, the fixed asset values of the peripheral districts are relatively high, and the post-disaster direct economic loss is higher in these districts. (2) BJ has a developed tertiary industry which accounts for $77.9 \%$ of the total GDP of the city. Affected by population and commercial distribution, the tertiary industry and its spatial distribution is mainly concentrated in the city center. 


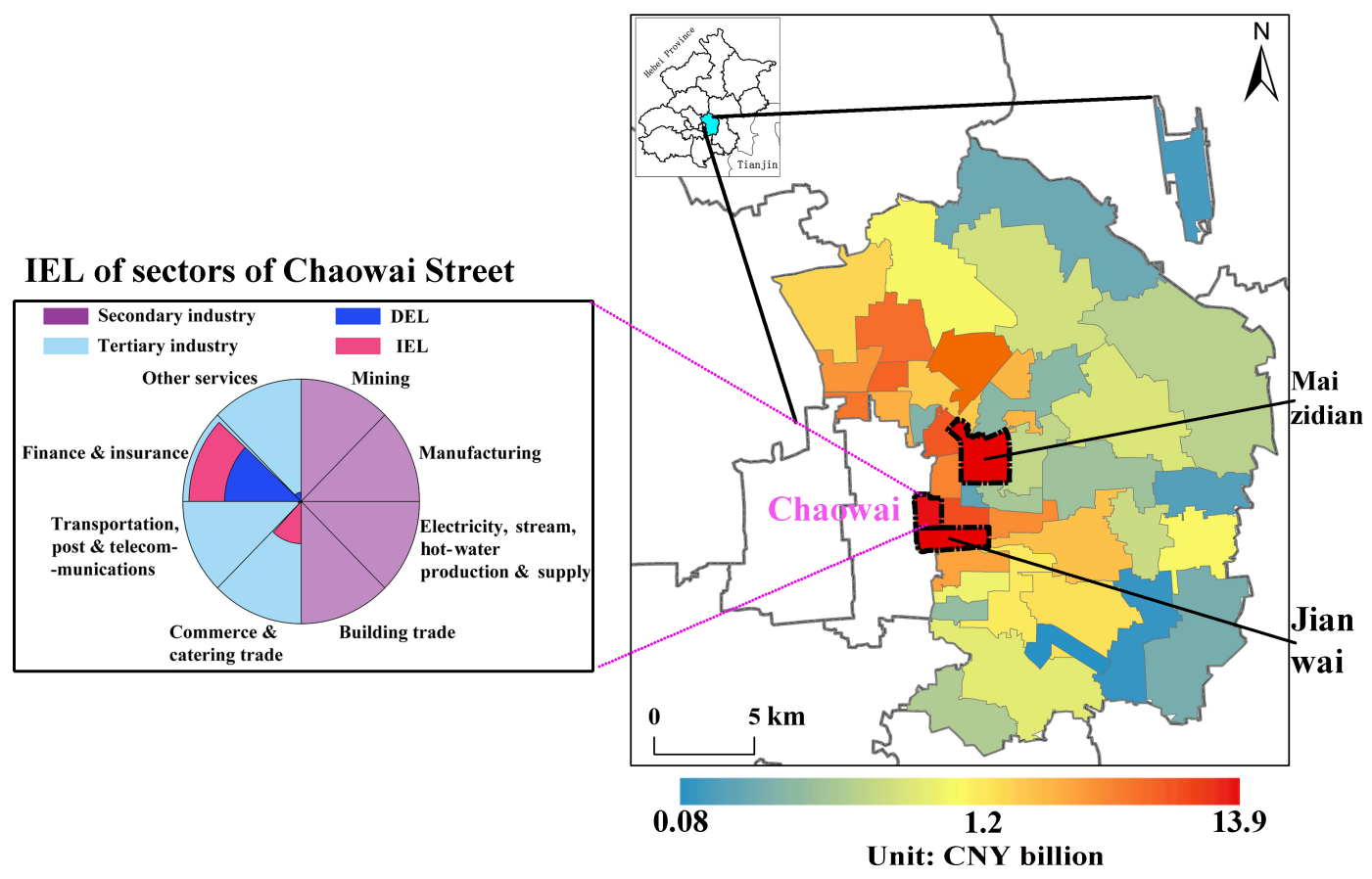

Figure 5. The right panel shows the ripple effects of the indirect economic loss (IEL) of the Chaoyang District; the left panel shows the direct (DEL) and indirect (IEL) economic loss of the sectors of the secondary industry and the tertiary industry of Chaowai Street.

\subsection{Analysis of the ripple effects of indirect economic loss and optimal recovery measures}

The Chaoyang District suffers the highest indirect economic loss, so the analysis is made by taking the Chaoyang District as an example. Areas with a high value in the Chaoyang District are mainly concentrated between the east 2 nd Ring Road and the east 3rd Ring Road; the three streets with the highest value are Chaowai Street (USD 1.6 billion), Jianwai Street (USD 1.5 billion), and Maizidian Street (USD 0.9 billion). Among them, the indirect economic loss of Chaowai Street is higher than its direct economic loss (USD 0.6 billion), 2.6 times that of its direct economic loss. According to the IRRE model, the reason for the high indirect economic loss of Chaowai Street can be further analyzed through its various sectors. The indirect economic loss of Chaowai Street is mainly focused on the tertiary industry such as the commerce and catering industry (14) and the finance and insurance industry (15) (Fig. 5). The indirect economic loss of the commerce and catering industry (14) is 39 times that of its direct economic loss and accounts for $25 \%$ of the total indirect economic loss of Chaowai Street. The indirect economic loss of the finance and insurance industry (15) of the street is 1.46 times that of its direct economic loss and accounts for $68 \%$ of the losses of the street. The Government should focus on these two sectors when making post-disaster recovery and reconstruction policies for Chaowai Street.

In terms of the optimal recovery measures for the commerce and catering industry (14), due to its small direct eco- nomic loss, its indirect economic loss is mainly caused by industrial linkage. Therefore, measures to increase market demands can be implemented to speed up its recovery. In terms of the optimal recovery measures for the finance and insurance industry (15), the government should consider the postdisaster recovery of the overall economic system. The severe damage to the fixed assets of the secondary industry leads to the seriously inadequate intermediate input and the decrease in demand from downstream industries leads to the decrease of production or the increase of inventory in the secondary industry. These may be the two major reasons that cause the increase in the indirect economic loss of the tertiary industry. When the government makes the recovery and reconstruction policy, we suggest that it not only to considers the impact of industrial linkage, but that it also promotes the postdisaster production capacity of the secondary industry, which can help BJ's economic system recover to the pre-disaster level. In addition, the industries distributed in the Chaoyang District are mainly tertiary industries, so the industrial structure of Chaoyang is also mainly dominated by the tertiary industry. When a great disaster occurs, the high loss value of this district will mainly be caused by the losses of the tertiary industry.

Besides, the manufacturing industry (automobile manufacturing) is mainly distributed in districts such as the Shunyi District; the industrial structure of this District is also mainly dominated by the secondary industry, so the majority of total losses in the Shunyi District are due to the secondary industry. Therefore, industrial distribution and industrial structure 


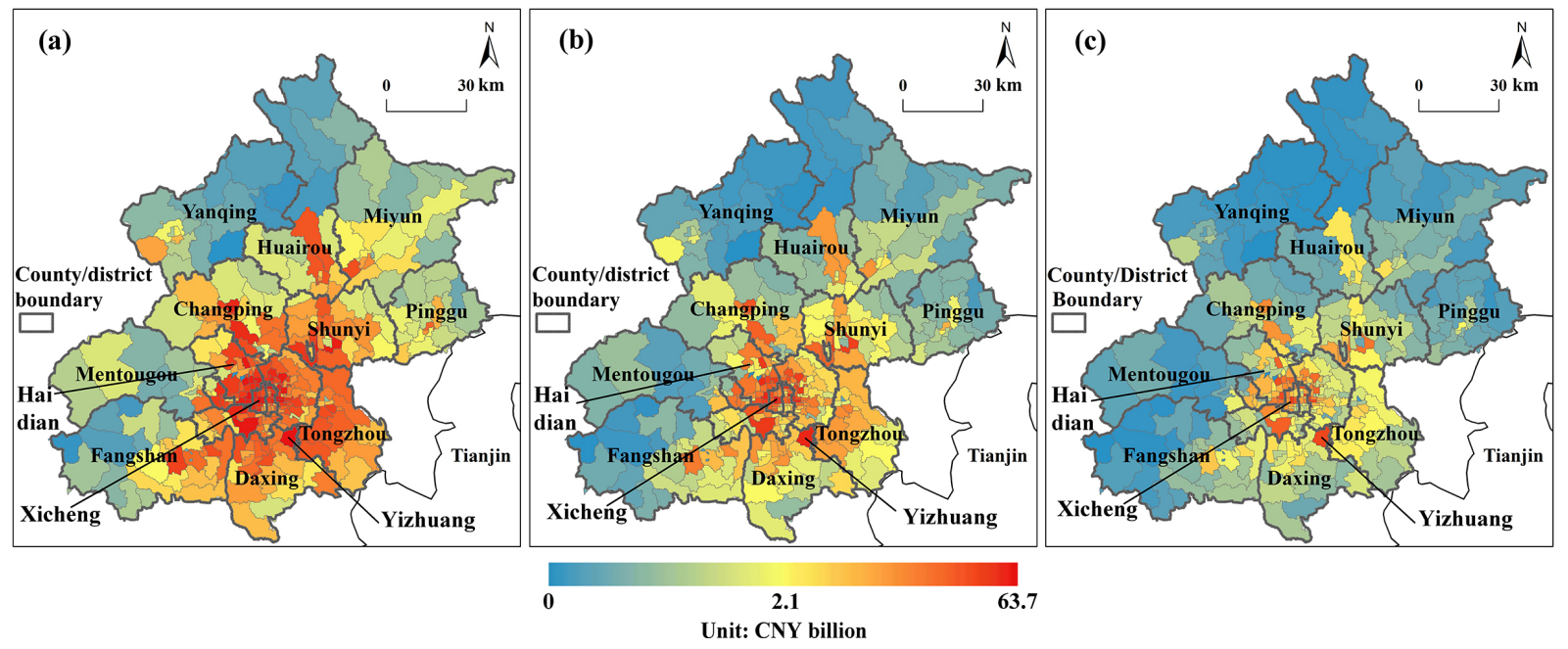

Figure 6. Evaluation results of the indirect economic loss of the streets, villages, and towns of Beijing (BJ) under the different rescue scenarios. (a) Scenario A; (b) Scenario B1; (c) Scenario C.

are key elements for determining the size of indirect economic loss of a region. In the course of post-disaster recovery and reconstruction, the government needs to take different recovery measures according to the industrial distributions and structures of the different regions.

By analyzing ripple effects, the overall loss can be spatially extended into each street and the sectors' losses in each street can be further evaluated, which helps government policy-makers intuitively get the information about the loss distribution and properly allocate rescue efforts when implementing recovery measures.

\subsection{The ripple effects under the different rescue scenarios}

The spatial distribution of the indirect economic loss under the three post-disaster rescue scenarios according to the IRRE model in BJ are illustrated in Fig. 6.

Under rescue scenario A, natural recovery, no rescue support will be given to the BJ's economic system, and its recovery will be accomplished by relying on its own economic structure and industrial linkage; therefore, its reconstruction and recovery period will be relatively longer with larger economic losses. It can be seen that the areas with high losses (Fig. 6a) are mainly distributed in the city center of BJ. The areas with the most severe losses are concentrated in the regions of the Xicheng District and the Haidian District, and they are extended to the regions of the Changping District, the Shunyi District, the Tongzhou District, and the Fangshan District. Under rescue effort scenario B (scenario B1: $120 \%, 6$ months), due to the introduction of external recovery funds and a series of recovery measures taken in the economic system such as the improvement in production capacity and substitution by the increase in imports, the overall loss is reduced. Spatially, the indirect economic loss of var- ious regions shows a decrease, and only some streets, villages, and towns see relatively high losses. The areas with high losses are mainly concentrated in city centers, such as in the Xicheng District and the Haidian District (Fig. 6b). Under rescue effort scenario $\mathrm{C}$, the losses of each region are further reduced, but the spatial heterogeneity shows an obvious difference between high value loss and low value loss (Fig. 6c). The high value regions like the Yizhuang region (including Economic Development Zone) still suffer losses without showing much decrease along with the improving rescue effort, while the losses of other regions are reduced significantly. Compared with the post-disaster affected area of rescue scenario A, the area of rescue scenario $\mathrm{C}$ is reduced by $4364 \mathrm{~km}^{2}$, accounting for $26.6 \%$ of the total area of BJ. This indicates that when the rescue effort is increased to $150 \%$ within 3 months, the fixed assets of various economic sectors can be recovered with the fastest speed, and the industrial linkage is established rapidly, which will help BJ's economic system recover faster and finish reconstruction.

The affected area calculation is based on disaster magnitude (Freckleton et al., 2012). According to the geometrical interval algorithm in GIS (geographic information system), the loss data of four scenarios are categorized into the five grades of catastrophe (USD 3.75 billion), disaster (USD 0.65 billion), medium disaster (USD 0.11 billion), small disaster (USD 0.019 billion), and micro disaster (USD 0.003 billion). The regions involved in the corresponding indirect economic losses of disasters with a grade higher than micro disaster are defined as the post-disaster affected areas. 


\section{Conclusion}

In order to reduce the long-term economic impacts caused by disasters and accelerate the economic recovery in the disaster aftermath, this paper uses two scenarios and three models to calculate and analyze the spatial heterogeneity of direct economic loss and the ripple effects of indirect economic loss in the streets, villages, and towns of BJ. We used the scenarios of direct economic loss to introduce the sectors' losses caused by the 2008 WCE in BJ, used the rescue scenarios to assess the necessity of improving rescue efforts, adopted the ARIO model to evaluate the indirect economic loss of BJ, and established the SDN model and the IRRE model to assess the direct and indirect economic loss of 321 streets, villages, and towns of BJ. The results indicate that the high value of indirect economic loss is more concentrated in the center of BJ compared with that of direct economic loss. The finance and insurance industry (15) of Chaowai Street in the Chaoyang District suffers the most serious indirect economic loss. During the 6 years after catastrophe occurred (20082014), the average annual GDP growth rate of BJ decreased from 8.55 to $4.91 \%$. Compared to BJ's $8 \%$ GDP growth rate target, it is a significant and noticeable economic impact.

Before the disaster, attention should be paid to the adjustment and optimization of the industrial structure. In the disaster emergency rescue stage, attention should be paid to the transportation and allocation of relief material. During the post-disaster recovery and reconstruction stage, attention should be paid and priority given to the development of the industries with a high industrial linkage coefficient in order to speed up the economic recovery. Therefore, the adjustment of industrial structures and the strengthening of industrial linkage can mitigate the impact of natural disasters. Besides, the optimization of the industrial structure and the close industrial linkage is of great significance in regional growth. In order to achieve the sustainable development of a region, a balance between economic development and disaster mitigation must be found. By taking it as a reference, a strategy for the optimization and the adjustment of the industrial structure should be established. These results can provide the scientific and effective aid to find the above mentioned balanced point to increase rescue effort and prioritize support for the industries which are located in the seriously damaged regions.

\section{Data availability}

The direct economic loss data are available from NCDR and MOST (2008); they will charge for the book. The inputoutput tables of the Beijing municipality and the Sichuan province are available upon request from the National $\mathrm{Bu}$ reau of Statistics of the People's Republic of China (Tel 01068576320) and is free of charge. The economic data of the streets, villages, and towns of the Beijing municipality are available upon request from the Beijing Macro Economic Social Development Basic Database (http://www.bjhgk.gov. cn/ww/MenuItemAction!queryMenu, Tel 4006507003); fees may be charged to apply for the license.

Competing interests. The authors declare that they have no conflict of interest.

Acknowledgements. The financial support was provided by the National Key Research and Development Program-Global Change Mitigation Project: Global change risk of population and economic system: mechanism and assessment (no. 2016YFA0602403); Beijing Municipal Natural Science Foundation (no. 9172010); Major Program of National Natural Science Foundation of China (no. 91325302); National Basic Program of China (973 Program, no. 2012CB955402); National Key R\&D Program (2016YFA0602604); National Natural Science Foundation of China (no. 41171401 and no. 41101506); and The Fundamental Research Funds for the Central Universities (no. 310421101).

Edited by: H. Kreibich

Reviewed by: two anonymous referees

\section{References}

Algermissen, S. T. and Steinbrugge, K. V.: Seismic Hazard and Risk Assessment: Some Case Studies, The Geneva Papers on Risk and Insurance, 9, 17, 1984.

Cochrane, H. C.: Forecasting the economic impact of a Midwest earthquake, University at Buffalo Institutional Repository MCEER Technical Reports, University at Buffalo, Buffalo, 223247, 1997.

Editorial department of Chinese geological education: Sichuan Wenchuan Earthquake and disaster relief, Chinese Geol. Educ., 17, 16-20, doi:10.16244/j.cnki.1006-9372.2008.02.006, 2008.

FEMA - Federal Emergency Management Agency: HAZUS 99 Estimated Annualized Earthquake Losses for the United States, Federal Emergency Management Agency, Washington, D.C., https://nehrpsearch.nist.gov/static/files/FEMA/PB2008108263. pdf (last access: March 2017), 20010

Freckleton, D., Heaslip, K., Louisell, W., and Collura, J.: Evaluation of transportation network resiliency with consideration for disaster magnitude, 91st annual meeting of the transportation research board, Washington, D.C., 2012.

Guivarch, C., Hallegatte, S., and Crassous, R.: The resilience of the Indian economy to rising oil prices as a validation test for a global energy-environment-economy CGE model, Energ Policy, 37, 4259-4266, doi:10.1016/j.enpol.2009.05.025, 2009.

Hall, R. and Jones, C.: Why Do Some Countries Produce So Much More Output per Worker than Others?, Quart. J. Econ., 114, 83116, doi:10.3386/w6564, 1999.

Hallegatte, S.: An adaptive regional input-output model and its application to the assessment of the economic cost of Katrina, Risk Anal., 28, 779-799, doi:10.1111/j.1539-6924.2008.01046.x, 2008 . 
Hallegatte, S.: Modeling the Role of Inventories and Heterogeneity in the Assessment of the Economic Costs of Natural Disasters, Risk Anal., 34, 152-167, doi:10.1111/risa.12090, 2014.

Hallegatte, S., Ranger, N., Mestre, O., Dumas, P., Corfee-Morlot, J., Herweijer, C., and Wood, R. M.: Assessing climate change impacts, sea level rise and storm surge risk in port cities: a case study on Copenhagen, Climatic Change, 104, 113-137, doi:10.1007/s10584-010-9978-3, 2010.

Hancilar, U., Tuzun, C., Yenidogan, C., and Erdik, M.: ELER software - a new tool for urban earthquake loss assessment, Nat. Hazards Earth Syst. Sci., 10, 2677-2696, doi:10.5194/nhess-102677-2010, 2010.

Jiren, Z. Z., Shifeng, H., Li, J. R., Ding, Z. X., Huang, S. F., and $\mathrm{Hu}$, Y. L.: Research of flood and waterlogging loss assessment model based on distribution social-economic database, J. China Inst. Water Resour. Hydropow. Res., 1, 104-110, 2003.

Li, J., Crawford Brown, D., Syddall, M., and Guan, D.: Modeling Imbalanced Economic Recovery Following a Natural Disaster Using Input-Output Analysis, Risk Anal., 33, 1908-1923, 2013.

Lijun, Y. Z., Weihua, H., Song, L. J., Zhang, Y., Yaer, Y. L., Hu, W. H., and Wang, J.: Discussion on the disaster assessment method of ensuing earthquakes, Inland Earthq., 12, 268-273, doi:10.16256/j.issn.1001-8956.1998.03.016, 1998.

Ming, J. L., Shuai, L., Tan, M., Sun, J., Li, S., Chen, J. B., Wu, C. Y., Yao, Y., Chang, X. D., Wang, Q., and Yiliyar, A.: Hazard investigation and analysis of the Yutian earthquake with MS 7.3 on February 12, 2014, Earthq. Res. China, 30, 233-242, doi:10.1001-4683(2014)02-0233-10, 2014.

Munich Re: Annual Review: Natural Catastrophes, Munich Reinsurance Company, Munich, 2002.

NCDR (National Commission for Disaster Reduction) and MOST: Wenchuan earthquake disaster - a comprehensive analysis and evaluation, Science Press, Beijing, 2008.

Okuyama, Y.: Economic modeling for disaster impact analysis: past, present, and future, Econ. Syst. Res., 19, 115-124, 2007.

Ranger, N., Hallegatte, S., Bhattacharya, S., Bachu, M., Priya, S., Dhore, K., Rafique, F., Mathur, P., Naville, N., Henriet, F., Herweijer, C., Pohit, S., and Corfee-Morlot, J.: An assessment of the potential impact of climate change on flood risk in Mumbai, Climatic Change, 104, 139-167, doi:10.1007/s10584-010-9979-2, 2010.
Rose, A.: Macroeconomic consequences of terrorist attacks: estimation for the analysis of policies and rules, in: BenefitCost Analyses for Security Policies, edited by: Mansfield, C. and Smith, V., Edward Elgar (EE), 172-200, doi:10.4337/9781784711085.00016, 2015.

Rose, A. and Liao, S.-Y.: Modeling Regional Economic Resilience to Disasters: A Computable General Equilibrium Analysis of Water Service Disruptions, J. Reg. Sci., 45, 75-112, doi:10.1111/j.0022-4146.2005.00365.x, 2005.

Rose, A., Benavides, J., Chang, S. E., Szczesniak, P., and Lim, D.: The regional economic impact of an earthquake: Direct and indirect effects of electricity lifeline disruptions, J. Reg. Sci., 37, 437-458, 1997.

Song, H.: Research on the New Urban Spatial Structure in Beijing City Master Planning, Urban Stud., 16, 36-40, 2009.

Tantala, M. W., Nordenson, G. J. P., Deodatis, G., and Jacob, K.: Earthquake loss estimation for the New York City Metropolitan Region, Soil Dynam. Earthq. Eng., 28, 812-835, doi:10.1016/j.soildyn.2007.10.012, 2008.

Wu, J., Li, N., Hallegatte, S., Shi, P., Hu, A., and Liu, X.: Regional indirect economic impact evaluation of the 2008 Wenchuan Earthquake, Environ. Earth Sci., 65, 161-172, doi:10.1007/s12665-011-1078-9, 2012.

Xia, Y., Guan, D., Jiang, X., Peng, L., Schroeder, H., and Zhang, Q.: Assessment of socioeconomic costs to China's air pollution, Atmos. Environ., 139, 147-156, doi:10.1016/j.atmosenv.2016.05.036, 2016.

Xie, W., Li, N., Wu, J. D., and Liu, X. Q.: Evaluation of indirect loss from hypothetical catastrophes in two regions with different economic development levels in China, Nat. Hazards Earth Syst Sci., 12, 3325-3335, doi:10.5194/nhess-12-3325-2012, 2012.

Xie, W., Li, N., Li, C., Wu, J.-D., Hu, A., and Hao, X.: Quantifying cascading effects triggered by disrupted transportation due to the Great 2008 Chinese Ice Storm: implications for disaster risk management, Nat. Hazards, 70, 337-352, doi:10.1007/s11069013-0813-9, 2013.

Zhou, F.-B., Wang, X.-X., and Liu, Y.-K.: Gas drainage efficiency: an input-output model for evaluating gas drainage projects, Nat. Hazards, 74, 989-1005, doi:10.1007/s11069-014-1224-2, 2014. 